\section{Investigation of Collapsed-cone Algorithm Accuracy in Small Fields and Heterogeneous Environments}

\author{
Razieh Zaghian ${ }^{1 \odot}$, Abdolazim Sedighi Pashaki², Abbas Hagh- \\ parast $^{3 *}{ }^{\odot}$, Mohammad Hadi Gholami ${ }^{4}$, Mahdi Mohammadi ${ }^{1}$
}

\begin{abstract}
Background: The use of small fields has increased by the emergence of advanced radiotherapy. Dose calculations of these fields are complex and challenging for many reasons such as lack of electrical equilibrium even in homogeneous environments, and this complexity will increase in presence of heterogeneity. According to the importance of delivery the accurate prescription dose to the target volume in the patient's body, the dose calculation accuracy of used commercial algorithms in clinical treatment planning systems (TPS) should be evaluated.

Objective: The present study aims to evaluate the accuracy of Collapsed-cone dose measurement algorithm in Isogray treatment planning system.

Material and Methods: In this analytical study, the measurements were made in tissue equivalent solid water phantom with lung and bone heterogeneities by Pinpoint dosimeter $\left(0.015 \mathrm{~cm}^{3}\right.$ sensitive volume $)$ in several radiation fields $(1 \times 1$ to $\left.5 \times 5 \mathrm{~cm}^{2}\right)$. The phantoms were irradiated with 6,10 and $18 \mathrm{MV}$ photon beams and finally, the results of experimental calculations were compared with treatment planning outputs.
\end{abstract}

Results: In all setups, the maximum deviation occurred in the field of $1 \times 1 \mathrm{~cm}^{2}$. Then, the maximum deviation was observed for $2 \times 2 \mathrm{~cm}^{2}$ field size; however, it was up to $5 \%$ for homogeneous water phantom and lung heterogeneity. In $3 \times 3 \mathrm{~cm}^{2}$ and larger fields, there was a good agreement between the results of the TPS and experimental dosimetry. The maximum deviation was observed in water-bone heterogeneity.

Conclusion: This algorithm was able to pass the standard audit criteria, but it is better to be used more cautiously in bone heterogeneity, especially in low energies.

Citation: Zaghian R, Sedighi Pashaki A, Haghparast A, Gholami MH, Mohammadi M. Investigation of Collapsed-cone Algorithm Accuracy in Small Fields and Heterogeneous Environments. J Biomed Phys Eng. 2021;11(2):143-150. doi: 10.31661/jbpe.v0i0.1121.

\section{Keywords}

Radiotherapy; Dosimetry; Small Field; Algorithms; Phantoms, Imaging; Absorbed Dose

\section{Introduction}

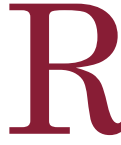
adiotherapy with ionizing radiation is one of the most frequent techniques for treating cancers [1,2]. Radiotherapy can lead to the DNA damage of cancer cells and stop cell dividing or growth, or prevent them from returning. Irradiation can also damage healthy cells, but the normal cells are more radiation-resistant. However, the
${ }^{1} \mathrm{MSc}$, Medical Physics Department, Students Research Committee,

Kermanshah University of Medical Sciences,

Kermanshah, Iran

${ }^{2} \mathrm{MD}$, Radiation oncolo-

gist, Faculty of Medicine,

Hamedan University

of Medical Sciences,

Hamedan, Iran

${ }^{3} \mathrm{PhD}$, Department of

Medical Physics, Faculty

of Medicine, Kerman-

shah University of Medi-

cal Sciences, Kerman-

shah, Iran

${ }^{4} \mathrm{PhD}$, Medical Physicist

of Radiotherapy, Mah-

dieh Radiotherapy and

Brachytherapy Charity

Center, Hamedan, Iran

*Corresponding author: Abbas Haghparast

Department of Medi-

cal Physics, Faculty of

Medicine, Kermanshah

University of Medical

Sciences, Kermanshah, Iran

E-mail: abbas.haghparast@gmail.com

Received: 22 January 2019 Accepted: 16 February 2019 
main goal of radiotherapy is to obtain an optimal balance between delivering the maximum dose to target volume and the minimum dose to normal tissue tumor [3]. To this end, the use of modern radiotherapy techniques such as intensity modulated radiotherapy (IMRT), stereotactic radiosurgery (SRS/SRT), and stereotactic body radiotherapy (SBRT) and as a result the use of small fields have increased [4].

The treatment targets in small fields $(\leq 4 \times 4$ $\mathrm{cm}^{2}$ ) are smaller than the size of the field in conventional radiotherapy. Therefore, target volume receives high radiation dose; on the other hand, smaller volume of healthy surrounding tissues has been exposed to radiation. Therefore, one of the most fundamental goals of radiation therapy is achieved; on the other hand, small-field dosimetry is a challenging issue for a variety of reasons that one of these reasons is lack of electron equilibrium intensifying the heterogeneity and accuracy of determination absorbed dose that becomes more complicated [5]. Electrical disequilibrium occurs when the range of secondary electrons is larger than field size, thus it can cause significant inaccuracies in absorbed doses in heterogeneous environments [6,7].

Delivering the accurate dose to treatment targets is an essential requirement in radiotherapy, as the criterion success rate of radiation therapy depends on the measurement of accurate absorbed dose in tumor and compliance with the amount of the prescribed dose. If the absorbed dose is less than prescribed dose, it can lead to the resistance of cancer cells to radiation or tumor recurrence. Conversely, if the absorbed dose be more than the prescribed dose, it can lead to death or serious damage to healthy surrounding tissues [7, 8]. According to international commission on radiation units and measurement (ICRU) reports, the accuracy of absorbed dose calculation required to control a tumor with less of complications is about $\pm 5 \%$ [8]. However, in the vicinity of the heterogeneous tissues, and especially in small fields, the dose distribution becomes more complicated and magnitude of the error increases $[1,4]$. Thus, prediction of the received dose in each part of the body is very important before initiating the radiotherapy $[9,10]$.

Calculations of absorbed dose are applied by TPSs. Basic of a TPS is algorithms of dose calculations $[11,12]$. The use of low accuracy algorithms can lead to prediction errors of the absorbed dose and inappropriate treatment since the accuracy of dose calculation in both small fields and heterogeneous environments leads to more errors in calculations; thus, TPS algorithms should be evaluated [4, 13].

The aim of present study was to evaluate the dose calculation accuracy of Collapsedcone algorithm (a model-based algorithm) of Isogray TPS in both homogeneous and nonhomogeneous media, for 6,10 and 18 MV photon beam energies in square small fields. The results of TPS calculation was compared with experimental results as a gold standard.

\section{Material and Methods}

This analytical study aims to determine the accuracy of Collapsed-cone dose calculation algorithm of Isogray TPS. For this purpose, cubic phantoms, which consisted of plates with dimensions of $30 \times 30 \mathrm{~cm}^{2}$ and $1 \mathrm{~cm}$ thickness, including soft tissue, bone, and lung equivalent materials (the setups is shown in Figure 1), were used. Electron density specifications and density of various types of used tissues in the present study are listed in Table 1.

Experimental measuring of absorbed doses at the interesting point in phantoms was studied by Pinpoint 31014 and Dose One electrometer (manufactured by PTW Germany and IBA respectively). This dosimeter is one of the type ionization chambers with $0.015 \mathrm{~cm}^{3}$ sensitive volume ( 0.1 and $0.5 \mathrm{~cm}$ of radius and length) and measuring the dose of high energy photons at high spatial resolution. This dosimeter is most effective in the following conditions: the range of gamma energy, cobalt -60 to 50 MV photons, temperatures of 10 to $40{ }^{\circ} \mathrm{C}$, and $2 \times 2$ to $30 \times 30 \mathrm{~cm}^{2}$ fields. This waterproof 


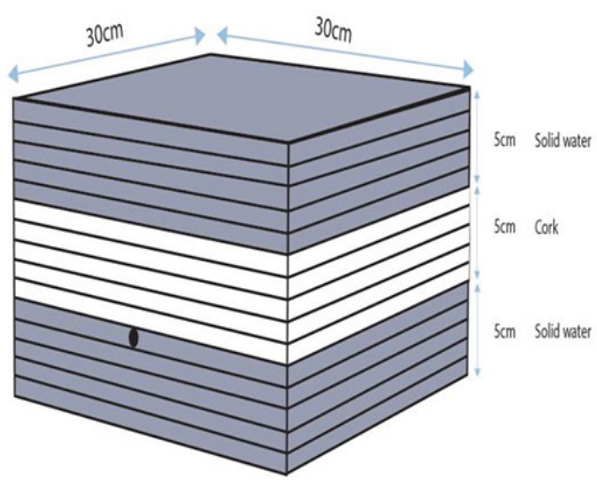

a)

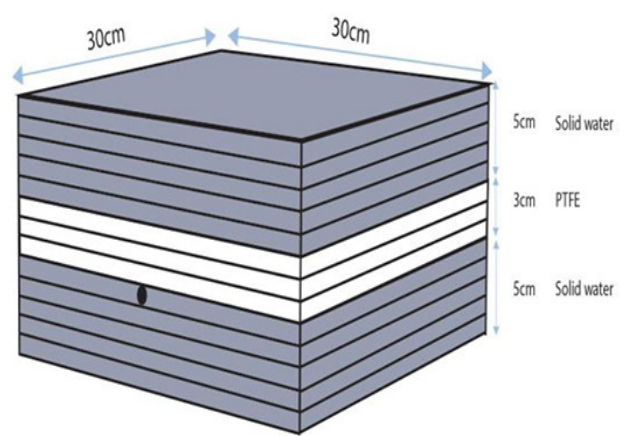

b)

Figure 1: Solid water phantom with a) lung and b) bone heterogeneity. The placement of dosimeter is shown as a small black circle.

Table 1: Specifications of different materials used in chest heterogeneous phantom.

\begin{tabular}{|c|c|c|c|}
\hline & Density $\left(\mathrm{g} / \mathrm{cm}^{3}\right)$ & $\begin{array}{l}\text { The electron density per } \\
\qquad \mathrm{cm}^{3} \times 10^{23}\end{array}$ & $\begin{array}{l}\text { Electron density relative to } \\
\text { water }\end{array}$ \\
\hline Soft-tissue & 1.06 & 3.48 & 1.042 \\
\hline SP34 & 1.045 & 3.539 & 1.003 \\
\hline lung & 0.21 & 0.69 & 0.207 \\
\hline Cork & 0.23 & 0.57 & 0.301 \\
\hline Bone & 2.00 & 5.03 & 1.506 \\
\hline PTFE & 2.2 & 6.243 & 1.868 \\
\hline
\end{tabular}

dosimeter can be used in air, water, and solid phantom with a polarity effect less than $2 \%$. The thickness of the sensitive pinpoint wall is $0.57 \mathrm{~mm}$ of PMMA with a density of 1.19 $\mathrm{g} / \mathrm{cm}^{3}$ and $0.90 \mathrm{~mm}$ graphite and a density of $1.85 \mathrm{~g} / \mathrm{cm}^{3}$. The central electrode is made of aluminum with a diameter of $0.3 \mathrm{~mm}$.

Radiation to the heterogeneous soft tissue-lung and soft tissuebone phantoms

In order to investigate the effect of soft tissue-lung heterogeneity, $5 \mathrm{~cm}$ Cork equivalent was sandwiched between $5 \mathrm{~cm}$ tissue equiva- lent material (solid water slabs) from top and $5 \mathrm{~cm}$ below (Figure 1a). To evaluate the inhomogeneity of soft tissue-bone, $5 \mathrm{~cm}$ solid water slabs were used in addition to $3 \mathrm{~cm}$ PTFE equivalent to bone and again $5 \mathrm{~cm}$ of solid water (Figure 1b). After heterogeneity, the Pinpoint dosimeter was placed at $0.5 \mathrm{~cm}$ in solid water slab (i.e.at 8.5 depth for the bone and $10.5 \mathrm{~cm}$ for the lung). The location of dosimeter is shown in Figure 2.

Then the phantoms were irradiated with an Elekta linear accelerator (Synergy) with three photon beams energies of 6,10 and $18 \mathrm{MV}$ and measurements made in radiation fields 
from $1 \times 1$ to $5 \times 5 \mathrm{~cm}^{2}$.

In all setups, the source to surface distance (SSD) was $100 \mathrm{~cm}$ and the gantry angle was $0^{\circ}$ so that the beam was perpendicular to the surface of the phantom and dosimeter.

The all of above steps were carried out for homogeneous solid water phantom and the absorbed dose was measured at depth of $5.5 \mathrm{~cm}$ (Figure 3).

\section{Dose calculation in TPS}

The slab phantoms with the same setups that are shown in Figures 1 and 3 were scanned using computed tomography (CT) scanner (16 slices) for treatment planning. After the CT simulation, transferring the sets of CT images to the TPS was performed. Then, 3D-CT datasets were reconstructed and the intended points were determined for dosimetry.

After calculating the absorbed dose by TPS, the results were compared with the results of the experimental dosimetry and the relative error percentage of $\mathrm{CC}$ algorithm was calculated according to the following formula.

Figure 2: The location of pinpoint is shown as a black line at the solid water slab's groove and in the center of it.

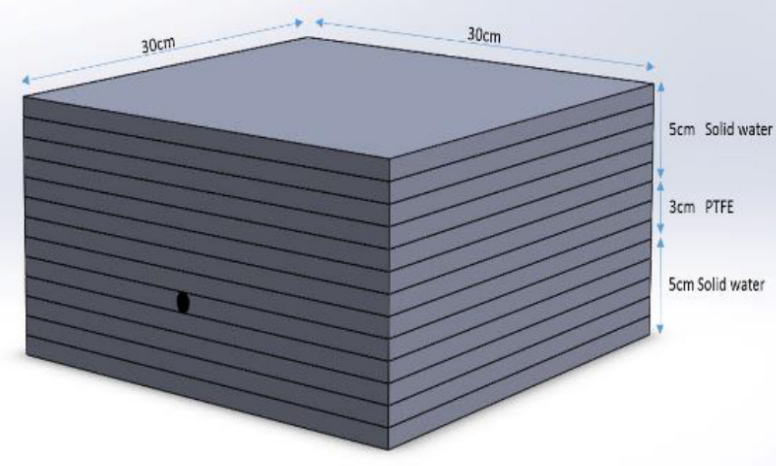

Figure 3: Solid water phantom (soft tissue equivalent). 


$$
\% E=\frac{\left(D_{M}-D_{T}\right)}{D_{M}} \times 100
$$

In which $D_{M}$ is calculated dose empirically and $D_{T}$ is the result of TPS calculations.

\section{Results}

Figure 4 illustrates the relationship between TPS relative error percentage and field size for three studied energies in all setups.

\section{Homogeneous water environment}

In this environment, for each of the 3 ener-

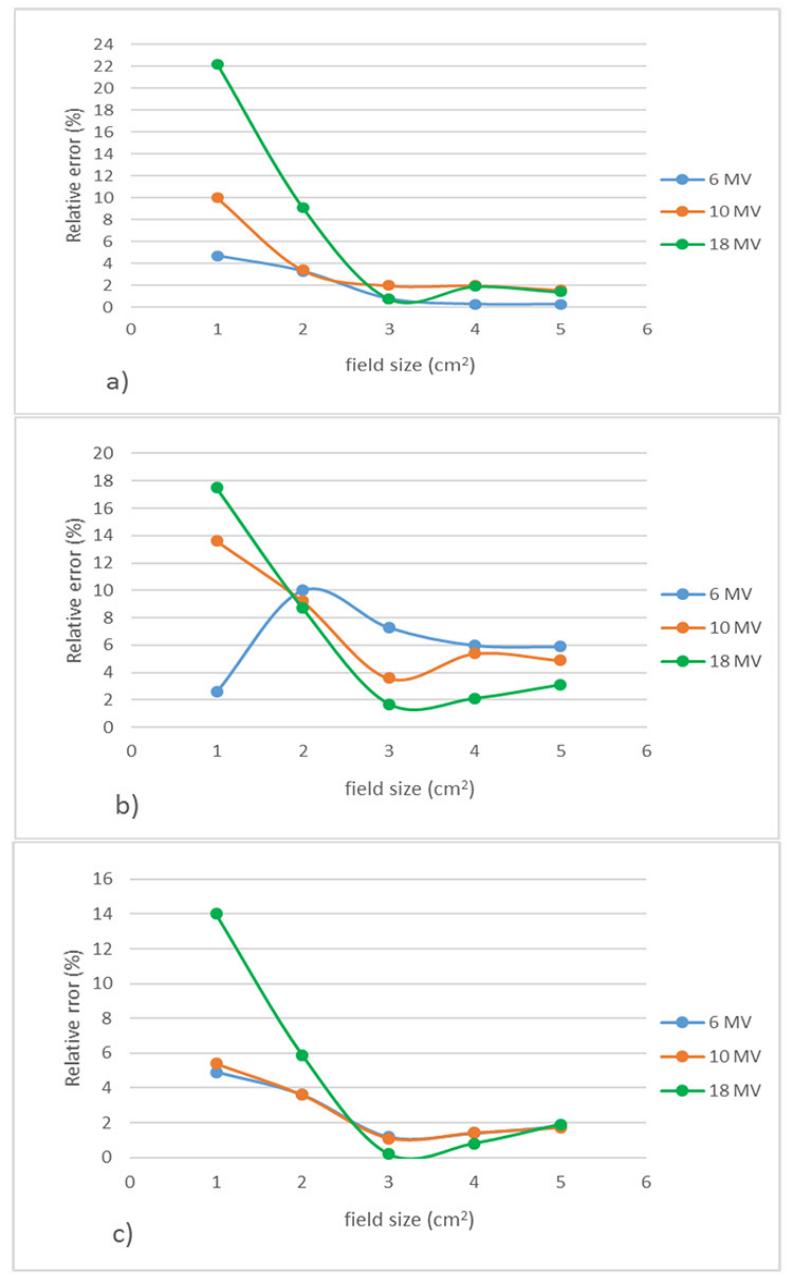

Figure 4: Curves of relative error percentage of calculated Collapsed-cone algorithm for every field size in three studied media a) solid water phantom with lung heterogeneity b) solid water phantom with bone heterogeneity and c) homogenous water phantom. gies studied, a significant decrease of deviation was observed with increasing field size to $3 \times 3$ $\mathrm{cm}^{2}$, but no significant change was observed for larger field sizes. Deviation increased with incremental energy in $1 \times 1 \mathrm{~cm}^{2}$. Equal to more than $2 \times 2 \mathrm{~cm}^{2}$ fields, there was no significant difference between the relative errors in different energies but founded the increasing magnitude of error from $10-18 \mathrm{MV}$ for $2 \times 2 \mathrm{~cm}^{2}$ field size. In the homogeneous water environment, at minimum and maximum deviations were $0.2 \%$ and $14 \%$ for energy $18 \mathrm{MV}$ and in $3 \times 3$ and $1 \times 1 \mathrm{~cm}^{2}$ field sizes, respectively. In the mentioned environment, deviation was negligible and up to $2 \%$ with the exception of $1 \times 1$ and $2 \times 2 \mathrm{~cm}^{2}$.

\section{Water/Lung heterogeneity}

The results obtained from this environment were similar to the homogeneous water media; thus, the deviation decreased with the increase of field size. But it increased in $18 \mathrm{MV}$ with increasing the field size from $3 \times 3$ to $4 \times 4 \mathrm{~cm}^{2}$. This increasing was still with an acceptable level of error. From another view, the energy dependence of the heterogeneity was similar to the homogeneous water phantom. In this environment, the minimum error occurred in $4 \times 4$ and $5 \times 5 \mathrm{~cm}^{2}$ field size with $6 \mathrm{MV}$ photon energy, and the maximum error occurred in $1 \times 1 \mathrm{~cm}^{2}$ field size with $18 \mathrm{MV}$ photon energy and their values were $0.03 \%$ and $22.2 \%$, respectively. In this environment, deviation was up to $2 \%$ with the exception of $1 \times 1$ and $2 \times 2$ $\mathrm{cm}^{2}$.

\section{Water/bone heterogeneity}

In this heterogeneity, which was compared to the previous two environments, there was a greater difference between the calculations of TPS and experimental measurements. The lowest and the highest deviation occurred in $3 \times 3 \mathrm{~cm}^{2}$ and $1 \times 1 \mathrm{~cm}^{2}$ fields for energy $18 \mathrm{MV}$ similar to the homogeneous water environment and deviations were $1.7 \%$ and $17.5 \%$, respectively. Although there was no signifi- 
cant difference in this energy for the magnitude of error in $3 \times 3 \mathrm{~cm}^{2}$ and larger fields, the minimum deviation occurred in the energy of $6 \mathrm{MV}(2.6 \%)$ in $1 \times 1 \mathrm{~cm}^{2}$ field size. Regardless of this data, deviation was reduced with increasing the field size, but there was no difference between $4 \times 4 \mathrm{~cm}^{2}$ and $5 \times 5 \mathrm{~cm}^{2}$ field sizes for $6 \mathrm{MV}$ photons. For energy of 10 and $18 \mathrm{MV}$, it decreased by increasing the size to $3 \times 3 \mathrm{~cm}^{2}$, and a slight increase was observed in larger field sizes than $3 \times 3 \mathrm{~cm}^{2}$. In all fields, a deviation reduction occurred with increasing energy.

\section{Discussion}

In this study, the accuracy of Collapsed-cone algorithm dose calculating from Isogray TPS was compared with the experimental results obtained using the pinpoint dosimeter in the homogeneous water phantom, heterogeneous water-lung and water-bone phantoms exposed to photon beam radiation with 6,10 and 18 MV in $1 \times 1$ to $5 \times 5 \mathrm{~cm}^{2}$ field sizes. In most similar studies, the Monte Carlo simulation has been used to verify the accuracy of dose calculation for clinical measurement algorithms $[5,14,15]$, although the Monte Carlo simulation can't be considered as a golden standard without experimental confirmation [16].

Due to the absence of a single protocol for absolute dose measurement in small fields, the complexity of direct measurement and comparing with calculations of the TPS, most of the studies have investigated the accuracy of algorithms with measuring the percentage of depth doses. The use of absolute dosimetry instead of the relative dosimetry for comparing the TPS results and also Pinpoint ionizing chamber (an ideal chamber for small field dosimetry) led into difference between this study from other studies.

Due to the challenges of small-field dosimetry, selecting a suitable detector for dose measurement in these fields is very necessary. According to study carried out by J. U. Wuerfel,et al., ionizing chambers are the best choice for measuring the absolute dose. The signal quantum noise of the dosimeters depends on the detector's material, in addition to the sensitive volume of the detector. This noise is very low in pinpoint dosimeter, even rather than diode type $E$ with a sensitive volume of $0.03 \mathrm{~mm} 3$. As a result, the precision and speed of the Pinpoint dosimeters are very high in dose measurement [17]. Furthermore, in a similar study carried out by Alagar [4], a scintillator plastic dosimeter was used, which has a high level of noise compared to chambers; the highest quantum noise can be expected from these detectors and dosimetry measurement is a timeconsuming performance.

This study was carried out in $1 \times 1$ to $5 \times 5 \mathrm{~cm}^{2}$ field sizes. For all setups, the greatest deviation was observed in the $1 \times 1 \mathrm{~cm}^{2}$ field size, except for bone heterogeneity at $6 \mathrm{MV}$ energy, which was consonant with the results of Alagar's study [4]. The maximum deviations of the $1 \times 1 \mathrm{~cm}^{2}$ field size for water-lung and water-bone heterogeneity were $22 \%$ and $17 \%$, respectively, and for the homogeneous water environment was $14 \%$. The most important reason for this significant error could be inappropriateness of the pinpoint dosimeter in field sizes smaller than $2 \times 2 \mathrm{~cm}^{2}$. The effect of charged particle disequilibrium in this small field should not be ignored.

Although the magnitude of error decreased significantly in $2 \times 2 \mathrm{~cm}^{2}$ field size, the final value was still significant. This behavior can be described based on the electrical disequilibrium effect. Deviation in this field for 6 and $10 \mathrm{MV}$ of photon energy in the lung and water heterogeneity was relatively lower and about $3.5 \%$. While for $18 \mathrm{MV}$ energy, it was $9.1 \%$ and $5.9 \%$ in the lungs and water, respectively. Due to the enhancement of the photon energy, the range of the secondary scatter electron has increased and the effect of electrical disequilibrium, which is usually not considered in the treatment planning algorithms, leads to more deviation.

Generally, for field sizes larger than $2 \times 2$ 
$\mathrm{cm}^{2}$, a significant reduction in deviation was observed with increasing field size in all setups; thus, there was a good agreement between experimental results and TPS calculations for $3 \times 3 \mathrm{~cm}^{2}$ and larger field sizes in all energies. (Deviation less than 2\% in water and lung and less than $5 \%$ in bone). Although the deviation is expected to be low in all fields for homogeneous water environment but it should be noted that the electrical disequilibrium effect exists in small fields and high energies, even in homogeneous environments. Thus, it seems this effect causes a relatively significant deviation in $1 \times 1$ and $2 \times 2 \mathrm{~cm}^{2}$ field sizes especially in $18 \mathrm{MV}$. On the other hand, it was expected that the deviation in heterogeneous lung environment is higher than homogeneous water; however, this difference was not observed except in the $1 \times 1 \mathrm{~cm}^{2}$ field size and in $18 \mathrm{MV}$ energy for a $2 \times 2 \mathrm{~cm}^{2}$ field size, which could be one of the defects of the studied algorithm.

Jones et al. [5] showed that in low-density medium such as a lung for a photon $6 \mathrm{MV}$ photon in $3 \times 3 \mathrm{~cm}^{2}$ and larger field sizes, the charged particle equilibrium can be recovered and the algorithms can fairly predict the dose in and out of the inhomogeneity. In the present study, it was also observed that there is an appropriate correlation between the calculations of the TPS and experimental measurements for this region and $6 \mathrm{MV}$ energy; thus, the magnitude of error was up to $1 \%$.

In the presence of high-density heterogeneity, such as bone, deviation was dropped by increasing the energy values, similar to the results of Alagar's study [4]. In this heterogeneity, the greatest deviation was observed in 6 $\mathrm{MV}$ for all fields except for $1 \times 1 \mathrm{~cm}^{2}$.

The dose calculation deviation of bone heterogeneity in each field size and energy was higher than other environments in contrast to the observation of Alagar. This phenomenon could be due to differences in type of dosimeter and algorithm; in addition, TPS could not calculate backscattering electrons effect on dose distribution in materials with high den- sity such as bone. On the other hand, due to the major difference between water and bone density and the considerable effect of heterogeneity in low energies, the heterogeneous correction seems to be inadequate in a Collapsed-cone algorithm.

\section{Conclusion}

Based on comparison between results of experimental measurement and calculation of absorbed dose by Collapsed-cone algorithm in a homogeneous water environment and also after inhomogeneous environments of lung and bone, the greatest deviation was occurred for the $1 \times 1 \mathrm{~cm}^{2}$ field size, except for the bone heterogeneity at $6 \mathrm{MV}$. According to this, the Pinpoint dosimeter is not ideal for the absorbed dose measurement in $1 \times 1 \mathrm{~cm}^{2}$ field size; thus, we cannot definitely comment on the accuracy or inaccuracy of the Collapsed-cone algorithm in this field size and this relatively large magnitude of deviation cannot be attributed separately to one of the two mentioned items.

In all setups, the magnitude of error decreased by increasing the field size, but there was no specific relationship between deviation and energy of the photons. In most of the obtained data, the Collapsed-cone algorithm has overestimation rather than experimental measurements. In the case of high dose calculation, overestimation values could lead to defective tumor treatment or recurrence. Although in this study, regardless of the $1 \times 1 \mathrm{~cm}^{2}$ field size, there was an appropriate accordance between the results of the experimental measurements and TPS calculations; however, the observed deviation in water-bone heterogeneity compared to the water-lung heterogeneity and the homogeneous water environment was significant especially in low energies due to the weakness of the Collapsed-cone algorithm in heterogeneous correction, or the inconsideration of the backscattering electrons effect in the presence of high-density materials. Nevertheless, it is better to use this algorithm with more caution in presence of inhomogeneity 
with high density (such as bone), and especially in low energies.

\section{Acknowledgment}

Authors gratefully acknowledge the Research committee of Kermanshah University of Medical Sciences for the financial support. Also, the authors declare their gratitude to staff of Radiotherapy Department of Imam Reza Hospital, Kermanshah, Iran and Mahdieh Radiotherapy and Brachytherapy Charity Center, Hamedan, Iran.

\section{Conflict of Interest}

None

\section{References}

1. Golestani A, Houshyari M, Mostaar A, Arfaie AJ. Evaluation of dose calculation algorithms of Isogray treatment planning system using measurement in heterogeneous phantom. Reports of Radiotherapy and Oncology. 2015;2(3):e5320. doi: 10.17795/rro-5320.

2. Falahati F, Nickfarjam A, Shabani M. A Feasibility Study of IMRT of Lung Cancer Using Gafchromic EBT3 Film. J Biomed Phys Eng. 2018;8:347-56. doi: 10.31661/jbpe.v0i0.791. PubMed PMID: 30568924. PubMed PMCID: PMC6280119.

3. Laub WU, Wong T. The volume effect of detectors in the dosimetry of small fields used in IMRT. Med Phys. 2003;30:341-7. doi: 10.1118/1.1544678. PubMed PMID: 12674234.

4. Alagar AG, Mani GK, Karunakaran K. Percentage depth dose calculation accuracy of model based algorithms in high energy photon small fields through heterogeneous media and comparison with plastic scintillator dosimetry. J Appl Clin Med Phys. 2016;17:132-42. doi: 10.1120/jacmp. v17i1.5773. PubMed PMID: 26894345. PubMed PMCID: PMC5690200.

5. Jones A0, Das IJ. Comparison of inhomogeneity correction algorithms in small photon fields. Med Phys. 2005;32:766-76. doi: 10.1118/1.1861154.

6 . Andreo P. The physics of small megavoltage photon beam dosimetry. Radiother Oncol. 2018;126:20513. doi: 10.1016/j.radonc.2017.11.001. PubMed PMID: 29191457.

7. Svensson GK. Quality assurance in radiation therapy: physics efforts. Int J Radiat Oncol Biol
Phys. 1984;10 Suppl 1:23-9.doi: 10.1016/03603016(84)90441-3. PubMed PMID: 6735791.

8. Wambersie A. The role of the ICRU in quality assurance in radiation therapy. Int $\mathrm{J}$ Radiat Oncol Biol Phys. 1984;10 Suppl 1:81-6. doi: 10.1016/03603016(84)90454-1. PubMed PMID: 6429103.

9. Fippel M. Fast Monte Carlo dose calculation for photon beams based on the VMC electron algorithm. Med Phys. 1999;26:1466-75. doi: 10.1118/1.598676. PubMed PMID: 10501045.

10. Jabbari K, Bagher Tavakoli M, Mojtaba Hosseini S. Development and Implementation of the Convolution Method for Photon Dose Calculation in Radiation Therapy. Journal of Isfahan Medical School. 2012;30(198):11.

11. Fogliata A, Nicolini G, Clivio A, Vanetti E, Cozzi L. Accuracy of Acuros XB and AAA dose calculation for small fields with reference to RapidArc $®$ stereotactic treatments. Med Phys. 2011;38:6228-37.

12. Singh N, Painuly NK, Chaudhari LN, Chairmadurai A, Verma T, Shrotiya D, et al. Evaluation of AAA and XVMC Algorithms for Dose Calculation in Lung Equivalent Heterogeneity in Photon Fields: A Comparison of Calculated Results with Measurements. $J$ Biomed Phys Eng. 2018;8:223-30. PubMed PMID: 30320026 . PubMed PMCID: PMC6169117.

13. Fogliata A, Lobefalo F, Reggiori G, Stravato A, Tomatis $S$, Scorsetti $M$, et al. Evaluation of the dose calculation accuracy for small fields defined by jaw or MLC for AAA and Acuros XB algorithms. Med Phys. 2016;43:5685. doi: 10.1118/1.4963219. PubMed PMID: 27782735.

14. Mesbahi A, Dadgar H. Dose calculations accuracy of TiGRT treatment planning system for small IMRT beamlets in heterogeneous lung phantom. Int J Radiat Res. 2015;13:345-54.

15. Han T, Mikell JK, Salehpour M, Mourtada F. Dosimetric comparison of Acuros XB deterministic radiation transport method with Monte Carlo and model-based convolution methods in heterogeneous media. Med Phys. 2011;38:2651-64. doi: 10.1118/1.3582690. PubMed PMID: 21776802. PubMed PMCID: PMC3107831.

16. Bagheri $H$, Soleimani $A$, Gharehaghaji N, Mesbahi A, Manouchehri F, Shekarchi B, et al. An overview on small-field dosimetry in photon beam radiotherapy: Developments and challenges. J Cancer Res Ther. 2017;13:175-85. doi: 10.4103/09731482.199444. PubMed PMID: 28643730.

17. Würfel JU. Dose measurements in small fields. Med Phys. 2013;1:81-90. 